\title{
Bioinspired elastic piezoelectric composites for high-performance mechanical energy harvesting
}

\author{
Q.Y. Zhu, H. Sun, Y. Zhang
}

School of Materials Science and Engineering, Wuhan University of Technology, 430070, Wuhan, China cglamri@whut.edu.cn

We report the sea sponge-inspired design and preparation of piezoelectric composite generators (PCGs) based on a three-dimensional electroceramic skeleton. Figure 1 shows the detailed fabrication process and the microstructure of obtained three-dimensional electroceramic skeleton. The remarkable improvements in the piezopotential of the bioinspired structure have been theoretically analyzed using numerical simulations based on a phase-field simulation, as illustrated by Figure 2. The open-circuit voltage, short-circuit current density and instantaneous power density of the bioinspired PCG reach up to $\sim 25 \mathrm{~V}, \sim 550 \mathrm{nA} / \mathrm{cm}^{2}$ and $\sim 2.6 \mathrm{~mW} / \mathrm{cm}^{2}$, respectively, corresponding to about 16 times higher power than that of conventional particle based piezoelectric polymer composites. Moreover, the bioinspired PCG displays 30 times higher strainvoltage efficiency under stretching than the state-of-the-art performance of the flexible piezoelectric energy harvesters reported so far.

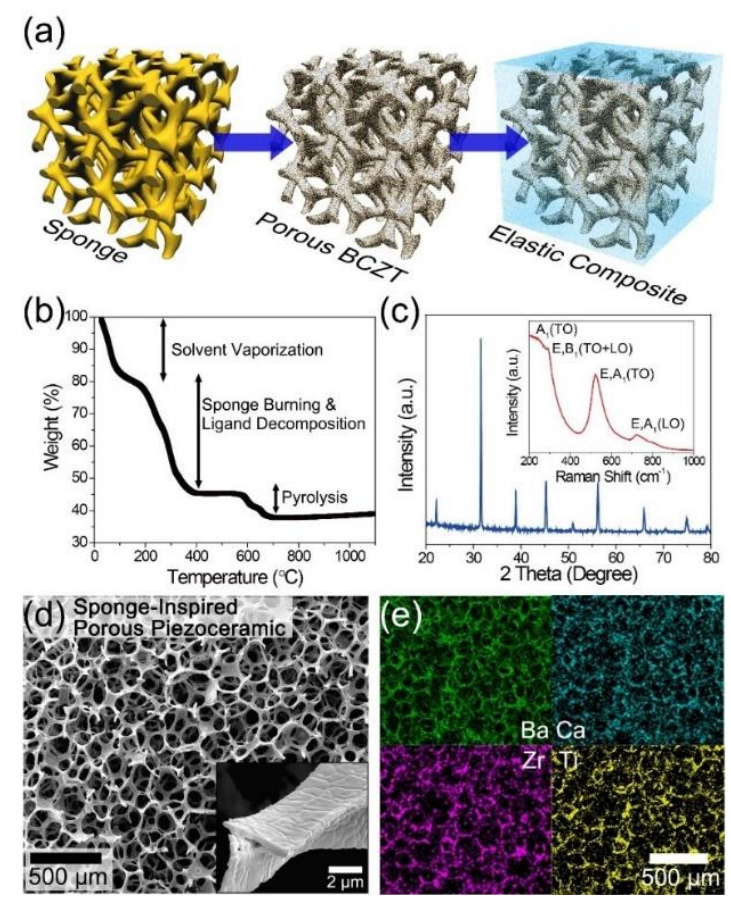

Figure 1. (a) Schematic of the fabrication process of the sea sponge-inspired 3D piezoelectric composite. (b) TGA curve during the thermal annealing and calcination. (c) XRD pattern and Raman spectrum (inset) of the bioinspired BCZT porous structure. (d) SEM image of BCZT porous structure. Inset: a magnified SEM image presenting the cross section of porous BCZT branch. (e) EDS mapping of BCZT porous structure. 


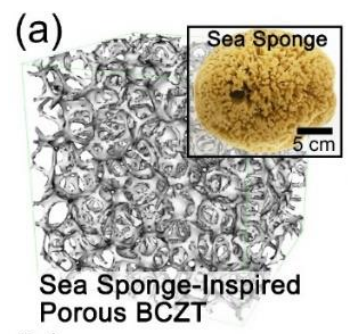

(b)

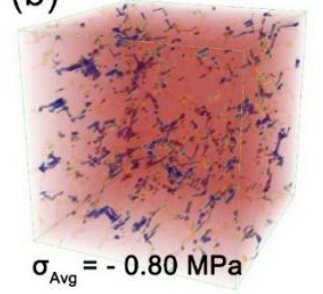

(c)

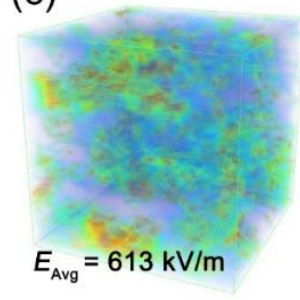

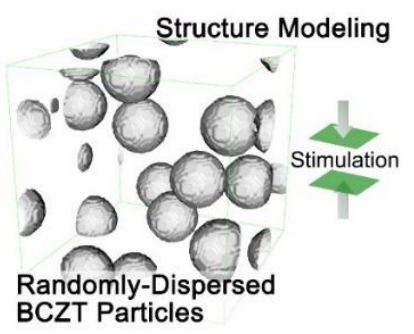
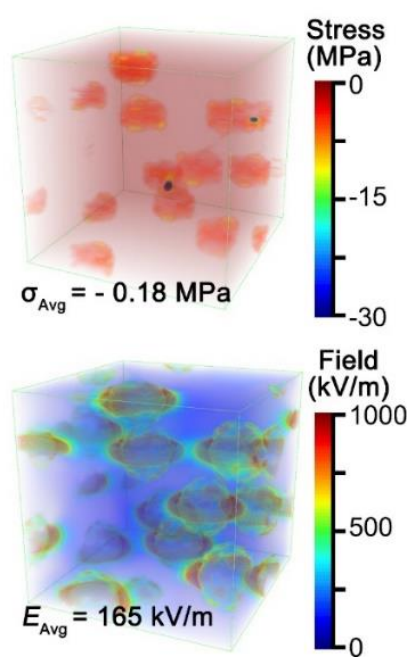

Figure 2. (a) Structure modelling of a sea sponge-inspired BCZT composite and a randomlydispersed BCZT particle-based composite by using the phase-field simulation with the Fourier spectral iterative perturbation method. (b) Computation results of the formed stress within the composites when a compressive strain of $12 \%$ is applied. (c) Computation results of the piezopotential-based electrical field generated within the composites when $12 \%$ strain is applied.
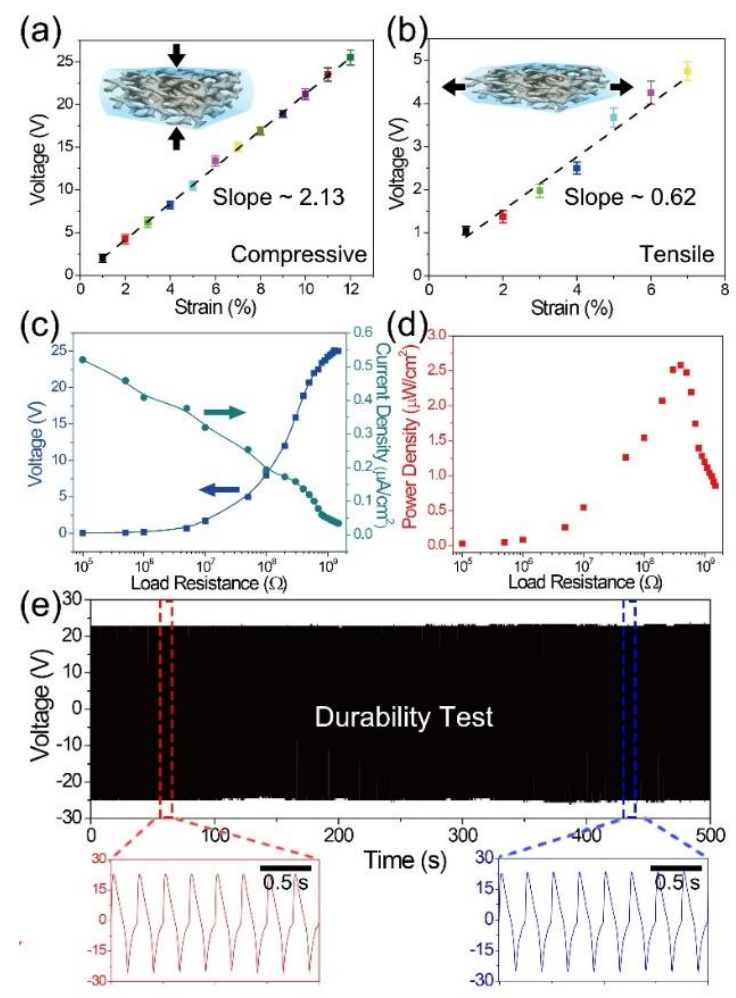

Figure 3. Strain-generated voltage of the elastic PCG according to (a) compressive and (b) tensile strain. (c) Output voltage, current density, and (d) instantaneous power density produced by the PCG according to circuital load resistors. (e) The voltage signals generated from the PCG in the cyclic test. 